\title{
Polarization disks in near-infrared high-resolution imaging
}

\author{
K. Murakawa
}

\author{
Max-Planck-Institut für Radioastronomie,Auf dem Hügel 69, 53121 Bonn, Germany \\ e-mail: murakawa@mpifr-bonn.mpg.de
}

Received 29 January 2010 / Accepted 29 April 2010

\section{ABSTRACT}

\begin{abstract}
A polarization disk is a characteristic feature of optical and near-infrared (NIR) polarimetric images of young stellar objects (YSOs) and is regarded as convincing evidence that a dust disk is present. We analyze high-resolution linear polarization maps of a sample of low-mass YSO disk models by means of radiative transfer calculations to investigate the effects of the disk geometry and grain sizes on polarization properties. Our modeling assumes spherical grains with a power-law size distribution of $n(a) \propto a^{-3.5} ; 0.005 \mu$ m $\leq$ $a \leq a_{\max }$ and with a fixed $a_{\max }$ of $0.25 \mu \mathrm{m}$ for the outer envelope and a different $a_{\max }$ for the disk. The parameters to examine are the disk height (i.e. the ratio of the disk height to the outer disk radius $H$ of 0.1 to 1.0 ) and the dust sizes in the disk (i.e. $a_{\max }$ of 0.25 to $1000.0 \mu \mathrm{m})$. In a near pole-on view, the polarization vectors are centro-symmetrically aligned even towards the disk, but the degree of polarization can be different from the envelope. We predict that the pole-on disk can be distinguished from the envelope. In contrast, the model images show a bipolar nebulosity and a polarization disk with a vector alignment in edge-on view. The polarization is low $(<10 \%)$ for large grains or low $H$ values and high (up to $\sim 80 \%$ ) for small grains and high $H$ values. In contrast, comparably constant polarizations (20-40\%) are obtained in the optical. The wavelength dependence in low NIR polarization cases is often detected in many $\mathrm{T}$ Tauri stars, suggesting that grain growth or an advanced disk accretion is expected in these objects. The opposite trend in high NIR polarization cases, which is found in some low-mass protostars, is reproduced with spherical grain models. To understand our results, we developed a generalized scattering model, which is an extension of the vector alignment mechanism. In the lowmass star disk case, multiple-scattered light behaves as if it chooses paths of comparably low optical density region (e.g. the disk surface), avoiding a high density, equatorial region, which we call the roundabout effect. The single-scattered light does not reach the observer, and the double-scattered light contributes the most flux. However, the effect of the first scattering still appears in the final polarization status. The higher the disk height in our models, the closer to $90^{\circ}$ the scattering angle on the disk surface, resulting in a higher polarization. The variety of the wavelength dependence on the polarization is also an example of the roundabout effect. In the optical, only stray light passed through the envelope reaches the observer. Thus, the optical polarization is characterized by scattering by small grains in the envelope. On the other hand, since the NIR photons can pass through a somewhat inner part of the disk, the NIR polarization can still offer information on the dust and geometry of the disk. We expect that a polarization disk analysis in high-resolution data, such as the one we present, offers opportunities to investigate the grain growth and dust settling in YSOs, and our new scattering model is also fundamental for nonspherical grain models.
\end{abstract}

Key words. circumstellar matter - dust, extinction

\section{Introduction}

Optical and near-infrared (NIR) imaging polarimetry is a powerful technique for investigating dust properties in the circumstellar environment. Many previous polarization maps of young stellar objects (YSOs) associated with a bipolar reflection nebula seen nearly edge-on show characteristic signatures of centrosymmetrically aligned polarization vectors in the nebulosity at large distances from the central star and a polarization disk with the vector alignment in the middle of the nebulosity. Bastien \& Ménard (1988, hereafter BM, 1990) explored the physics of polarization disk for the first time. The background of their work is an interpretation of the alignment of the polarization orientations in the polarization disk.

At that time, it was widely believed that the vector alignment comes from dichroic extinction of nonspherical grains that are aligned with a toroidal magnetic field in the disk (e.g. WarrenSmith et al. 1987). However, BM point out that it is difficult to explain some observational results with dichroism. For example, high polarizations (e.g. $\gtrsim 10 \%$ ) are often detected, and the degree of linear polarization and its orientation vary much $(\Delta P \geq 1 \%$ or $\Delta \theta \geq 30^{\circ}$ ) and rapidly (daily) in some objects. In addition, the magnetic structure in the disk is still controversial. Some theoretical works predict that a poloidal field is possible (Mestel \& Paris 1984; Königl 1991; Ostriker \& Shu 1995). Thereupon, BM introduced an alternative model of vector alignment. The idea is that the light emitted from the central star scatters first on the surface of the flat disk and then on the near edge of the disk. Their explanation proves that the alignment is reproduced even without aligned nonspherical grains, i.e. with only spherical grains, and can explain many observed linear polarization maps.

After a while, a trend began for modeling the imaging polarimetry by applying a disk and an infalling envelope geometry to explain the polarization maps (Kenyon et al. 1993; Whitney \& Hartman 1993; Fischer et al. 1994, 1996; Whitney et al. 1997; Lucas \& Roche 1997, 1998; Wood et al. 1998). Their models result in an $\sim 100$ AU centrifugal radius that reproduced polarization maps well, compared to the observations of many lowmass $\mathrm{T}$ Tauri stars. Their estimates of the centrifugal radius is in good agreement with the disk radii obtained in submillimeter and millimeter observations, although the polarization disk does not directly indicate a real disk (a structure with rotating matter inside the centrifugal radius) in general. Owing to the immense works by them and BM, it is now widely considered that 
a detection of a polarization disk is convincing evidence for the presence of the disk. However, some unresolved puzzles still remain. For example, the vector alignment pattern in models with only spherical grains sometimes does not fit the observations in detail very well. Another problem is that the NIR polarization increases or is nearly constant with increasing wavelength in some protostars, which is opposite in many T Tauri stars (e.g. Lucas \& Roche 1998).

It is quite natural to take the dichroism of aligned nonspherical grains (e.g. Whitney \& Wolff 2002; Wolf et al. 2002; Lucas et al. 2004; Beckford et al. 2008) into account. On the other hand, Piirola et al. (1992) and Whitney et al. (1997) have pointed out that a pseudo polarization pattern is produced if the disk is not spatially resolved. Unfortunately, all observations at the time suffered from this effect. In it, highly polarized scattered light from the nebulosity contaminates the flux from the disk region owing to an extended point-spread function (PSF). As a result, the polarization properties often echo that of the nebulosity. This causes a difficulty and a nuisance in interpreting and analyzing polarization data.

Recent NIR high-resolution imagers on the ground-based large telescopes and the space telescopes provide angular resolutions of $\sim 100$ mas. In principle, these instruments can spatially resolve disks in some favorable conditions, such as typical $\mathrm{T}$ Tauri stars with a disk radius of $100 \mathrm{AU}$ in a nearby star-forming region at a distance of $140 \mathrm{pc}$, which gives an angular disk size of 700 mas. Thus, the effect of an extended PSF ought to be relieved somewhat, and more direct investigation of the disk is possible. In fact, many demonstrative results of various object classes have been presented in recent publications (e.g. Meakin et al. 2005; Fujiyoshi et al. 2008; Jian et al. 2008; Murakawa et al. 2008a,c; Perrin et al. 2009; Simpson et al. 2009). In this paper, we evaluate a sample of low-mass YSO disk models by means of radiative transfer calculations of scattered light interacting with spherical grains. The goal is to explore a prescription for investigating the effects of the disk geometry and grain properties on the polarization disk in high-resolution images. To understand the model results, we revisit the BM mechanism to develop a generalized theory.

\section{Low-mass YSO disk model}

\subsection{Model assumption}

As is predicted from the present star formation theories, lowmass star models have an infalling envelope at a large distance from the central star and a rotating disk inside the envelope (Shakura \& Sunyaev 1973; Ulrich 1976; Cassen \& Moosman 1981; Pringle 1981; Terebey et al. 1984). We assume a form that is often used in T Tauri models, and the mass density distribution $\rho$ is given by

$$
\begin{aligned}
\rho & =\rho_{\mathrm{disk}}+\rho_{\mathrm{env}} \\
\rho_{\mathrm{disk}}(r, z) & =\rho_{\mathrm{d}}\left(r / R_{\mathrm{disk}}\right)^{-15 / 8} \exp \left(-\frac{\pi}{4}\left[\frac{z}{h(r)}\right]^{2}\right) \\
h(r) & =R_{\mathrm{disk}} H\left(r / R_{\mathrm{disk}}\right)^{9 / 8} \\
\rho_{\mathrm{env}}(R, \mu) & =\frac{\dot{M}_{\mathrm{env}}}{4 \pi \sqrt{G M_{\star} R^{3}}}\left(1+\frac{\mu}{\mu_{0}}\right)^{-1 / 2}\left(\frac{\mu}{\mu_{0}}+\frac{2 \mu_{0}^{2} R_{\mathrm{c}}}{R}\right)^{-1},
\end{aligned}
$$

where $r, z, R$, and $\mu$ are the two-dimensional cylindrical $(r, z)$ and spherical coordinates $\left(R=\sqrt{r^{2}+z^{2}}, \theta=\cos ^{-1} \mu\right)$. The function $h(r)$ indicates the disk height, and the value $H$ denotes the ratio
Table 1. Parameters of the modeled low-mass star disks.

\begin{tabular}{ll}
\hline \hline Parameters & Adopted values \\
\hline \multicolumn{2}{c}{ stellar parameter } \\
$T_{\star}$ & $4000 \mathrm{~K}$ \\
$L_{\star}$ & $1 L_{\odot}$ \\
$M_{\star}$ & $0.5 M_{\odot}$ \\
$d$ & $140 \mathrm{pc}$ \\
$R_{\star}$ & $1.45 \times 10^{11} \mathrm{~cm}$ \\
\hline \multicolumn{2}{c}{ dust grains (common) } \\
chemistry & DL-chemistry \\
size & $0.005 \leq a \leq a_{\max } \mu \mathrm{m}$ \\
\multicolumn{2}{c}{$\quad n(a) \propto a^{-3.5}$} \\
\hline$R_{\text {in }}$ & $0.05 \mathrm{AU}$ \\
$R_{\text {disk }}$ & $100 \mathrm{AU}$ \\
$M_{\text {disk }}$ & $0.01 M_{\odot}$ \\
$H$ & $0.1,0.2,0.3,0.5$, and 1.0 \\
$a_{\max }$ & $0.25,0.5,1.0,10.0,100.0$, and $1000.0 \mu \mathrm{m}$ \\
\hline$R_{\text {out }}$ & $5000 \mathrm{AU}$ \\
$\dot{M}_{\text {env }}$ & $1.0 \times 10^{-6} M_{\odot} \mathrm{yr}^{-1}$ \\
$\mu_{0}$ & $\cos \left(80^{\circ} / 2\right)$ \\
$a_{\max }$ & $0.25 \mu \mathrm{m}$ \\
\hline
\end{tabular}

of the disk height at the disk boundary to the disk outer radius. The mass density coefficient $\rho_{\mathrm{d}}$ is determined with the disk mass $M_{\text {disk }}$ by assuming a gas-to-dust mass ratio of 100 . The parameter $\mu_{0}$ is the angle of a stream line as related through the equation $\mu_{0}^{3}+\mu_{0}\left(R / R_{\mathrm{c}}-1\right)-\mu\left(R / R_{\mathrm{c}}\right)=0$.

The polar cavity is the region for $\mu>\mu_{0}$. The density inside the cavity is reduced to 0.01 compared to the surrounding envelope. The quantities $G$ and $R_{\mathrm{c}}$ are the gravitational constant and the centrifugal radius, respectively. The $R_{\mathrm{c}}$ is set to be $R_{\text {disk }}$. The (dust) disk is assumed to be in the region between the dust sublimation radius $R_{\text {in }}$ and the outer disk radius $R_{\text {disk }}$, and the infalling envelope is between $R_{\text {disk }}$ and the outer boundary $R_{\text {out }}$. For the stellar parameters, we refer to the ones presented in previous papers (e.g. Beckwith et al. 1990; Whitney et al. 2003; Andrews $\&$ Williams 2007). The adopted values are listed in Table 1. In these parameters, we are interested in the effect of the disk height in terms of the polarization disk property. In addition, the disk height is an important indicator of the dust settling. Thus, all parameters except the $H$ are fixed, and we examined the $H$ values of $0.1,0.2,0.3,0.5$, and 1.0 .

For dust grains, we assume spherical grains with an MRNlike power law size distribution (Mathis, et al. 1977); $0.005 \mu \mathrm{m} \leq$ $a \leq a_{\max } \mu \mathrm{m}$ and $n(a) \propto a^{-3.5}$. Since we are interested in the grain size effects but not the dependence of the chemical composition, we apply the DL-chemistry (Draine \& Lee 1984) often used as a stereotype of the circumstellar dust. The free parameter is only the $a_{\max }$. In our modeling, we assume different dust models, i.e. different $a_{\max }$ values, for the disk and the envelope. For the envelope, we adopted an interstellar grain population of $a_{\max }=0.25 \mu \mathrm{m}$. For the disk, we examined the models with an $a_{\max }$ of $0.25,0.5,1.0,10.0,100.0$, and $1000.0 \mu \mathrm{m}$. Figure 1 shows the scattering properties of the modeled dust. The polarization $P$ is calculated by $P=-S_{12} / S_{11}$. The quantities $S_{11}$ and $S_{12}$ are the scattering matrix elements, which are functions of the wavelength $\lambda$ and the scattering angle $\theta$ (e.g. Bohren \& Huffman 1983) and corresponds to the the Stokes $I$ and $Q$ parameters, respectively, just after the single scattering of the unpolarized light. Figure 1a shows the scattering angle dependence of the polarization at a wavelength of $2.2 \mu \mathrm{m}$. The scattering angle of the 

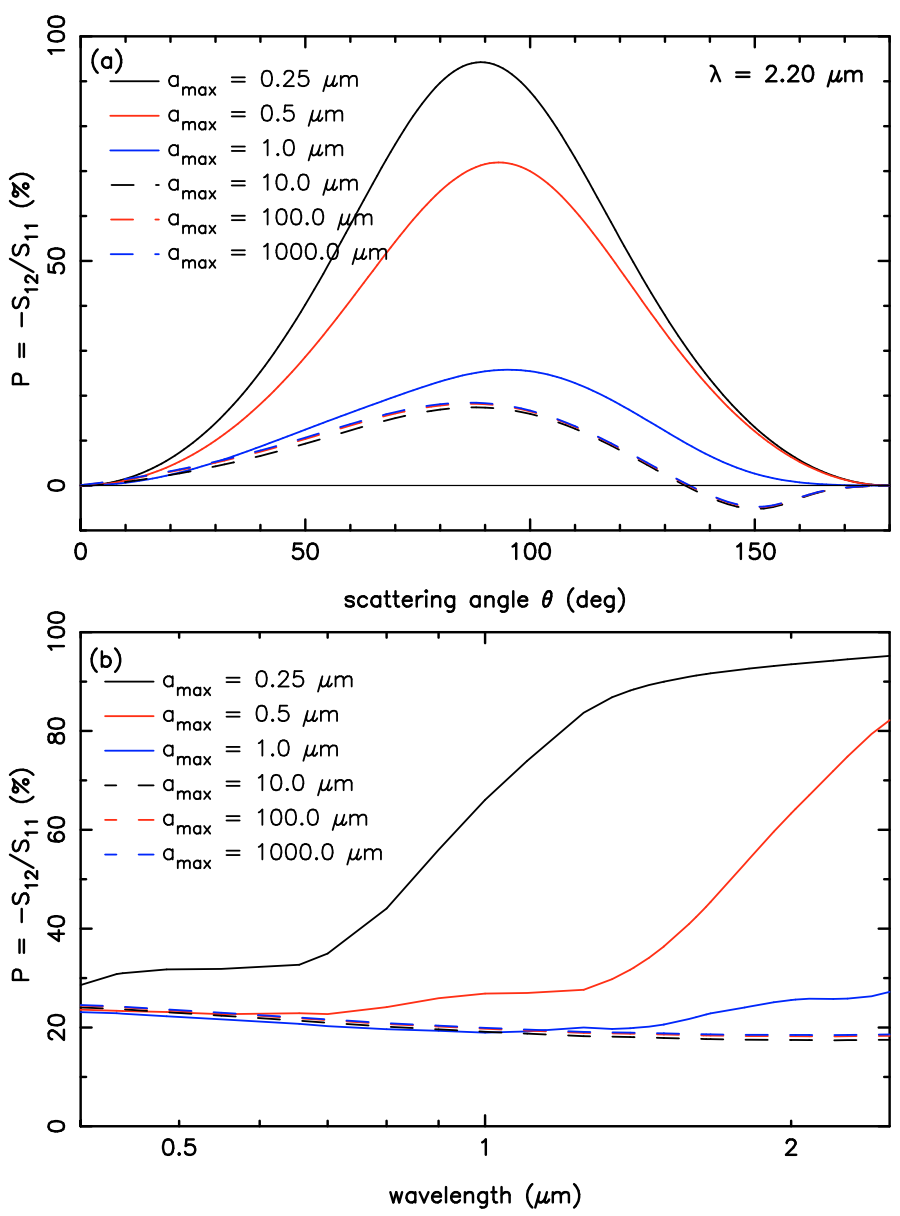

Fig. 1. The scattering properties of the modeled dust. The scattering angle dependence at a wavelength of $2.2 \mu \mathrm{m}$ a) and the wavelength dependence $\mathbf{b}$ ) on the polarization. The polarization $P$ is defined to be $P=-S_{12} / S_{11}$, where $S_{11}$ and $S_{12}$ are the scattering matrix elements. The results with different grain sizes are indicated with different line attributes.

maximum polarization $\theta_{\max }$ is around $90^{\circ}$ in all results. The polarizations are higher in smaller dust models. A size dependence is strong in small grains of $a_{\max } \lesssim 1 \mu \mathrm{m}$. On the other hand, it is weak for $a_{\max } \gtrsim 1 \mu \mathrm{m}$. In such grain sizes, the $P$ value becomes negative at large scattering angles $\left(\theta \gtrsim 135^{\circ}\right)$. This phenomenon is called polarization reversal, where the polarization orientation flips by $90^{\circ}$ (Bastien \& Ménard 1988; Fischer et al. 1994). Figure $1 \mathrm{~b}$ shows the wavelength dependence of $P\left(\theta_{\max }\right)$. In small dust models, the polarization increases with an increasing wavelength, and a large variation from $\sim 20 \%$ to $\sim 95 \%$ is seen in the NIR. In contrast, almost constant values of $\sim 20 \%$ are obtained for large grains. The above results demonstrate that the scattering properties strongly depend on the grain size in the small grain regime, where $x=2 \pi a / \lambda \lesssim 1$, but are insensitive in the large grain regime $(x \gtrsim 1)$.

\subsection{Effects of the disk height and the grain size}

With the aforementioned assumptions, we produced polarization images of the 30 parameter sets in the optical and NIR using our own Monte Carlo STSH code (Murakawa et al. 2008b). This code can treat multiple dust models for different regions in the model geometry and can compute the SED, the dust temperature, and the Stokes IQUV images. In these calculations, photons interacted with dust always scatter. Rather than simulating actual absorption, the photon weight is reduced at each interaction by a factor of $1-\omega$, where the value $\omega$ is the dust albedo. We produced images with different inclination angles $\theta_{\text {inc }}$ of $85^{\circ}$ (edgeon), $60^{\circ}, 30^{\circ}$, and $5^{\circ}$ (pole-on) at the same time to also see the effect of inclination angle. The resulting Monte Carlo images are convolved with a Gaussian function with a $0 . ' 1$ full-width at half maximum $(F W H M)$ size, which is a typical beam size in the NIR imaging obtained using the $8 \mathrm{~m}$ class telescopes and is smaller than the angular disk radius of 0.7 .

Figure 2 presents the polarization maps overlaid with the Stokes $I$ contour map. The wavelength is $2.20 \mu \mathrm{m}$. The upper panels are the results with an $a_{\max }$ of $0.25 \mu \mathrm{m}$ and $H$ of $0.1,0.3$, and 1.0 from left to right. In the bottom panels, the results with an $H$ of 0.3 and $a_{\max }$ of $1.0,10.0$, and $1000.0 \mu \mathrm{m}$ are presented. In these cases, the $\theta_{\text {inc }}$ is $85^{\circ}$. The resulting images show bipolar nebulosities and dark lanes bisepting the nebulosities. Except in the $H=0.1$ case, the two intensity peaks near the central star are sufficiently separated compared to the beam size, and a polarization disk with a horizontal vector alignment is seen. The polarization properties towards the polarization disk probably possess some information on the dust and density in these maps. This is, of course, the benefit of high-resolution imaging. The thickness of the dark lane increases with an increasing $H$ value. In a similar manner, the thickness of the polarization disk also increases. The horizontal extension of a polarization disk can be larger or smaller than the real dust disk depending on the model parameters, although it is not the case in the thickness (vertical extension). This equatorial extension also depends on the grain size. The smaller the grain sizes, the larger it appears. The degree of polarization depends on both the grain sizes and the disk height. The larger the grain sizes, the lower the polarization. We plot the polarization values as a function of the grain size $a_{\max }$ in Fig. 3 to see a general tendency. The polarization is derived by aperture polarimetry with a 0.2 circular diameter towards the central star. The wavelength is $2.20 \mu \mathrm{m}$. The polarization varies up to $\sim 80 \%$ and is high for a high $H$ value and a small grain size.

Figure 4 shows the effect of inclination angle. From left to right, $\theta_{\text {inc }}$ is $60^{\circ}, 30^{\circ}$, and $5^{\circ}$, cf. Fig. 2 for the edge-on case. The parameters $\left(H, a_{\max }\right)$ are $(0.3,0.25),(0.3,1000.0)$, and $(0.1,1000.0)$ from top to bottom. In the edge-on case, the real dust disk causes a reduction in the degree of polarization. In contrast, the dust disk behaves as a strong scattering body when the disk surface is directly seen and emits single-scattered light. For $\theta_{\text {inc }}=60^{\circ}$ and $30^{\circ}$, the appearance of the polarization patterns is different for the upper and lower sides, where the lower side is tilted towards the observer. In this geometrical configuration of the central star, the disk surface, and the observer, the scattering angle on the upper side is closer to $\theta_{\max }$. Thus, the polarization on this side is higher than the other side. When $\theta_{\text {inc }}$ is smaller, the vector pattern towards the disk becomes more centro-symmetric, as is seen in the outer envelope. These polarization properties are seen in previous similar modeling results (e.g. Whitney \& Hartman 1993; Fischer et al. 1996). Compared to the results with various geometry parameters, the difference in $H$ appears in the thickness of the equatorially extended regions with low polarizations, and the polarization values depend on the grain size in the disk. The latter is particularly interesting because this result implies that pole-on polarimetric images are potential laboratories for detecting regions where large grains exist and are a part of a real disk. Although no result has been reported with this method so far, such observations would only be possible if the strong unpolarized light from the central star 

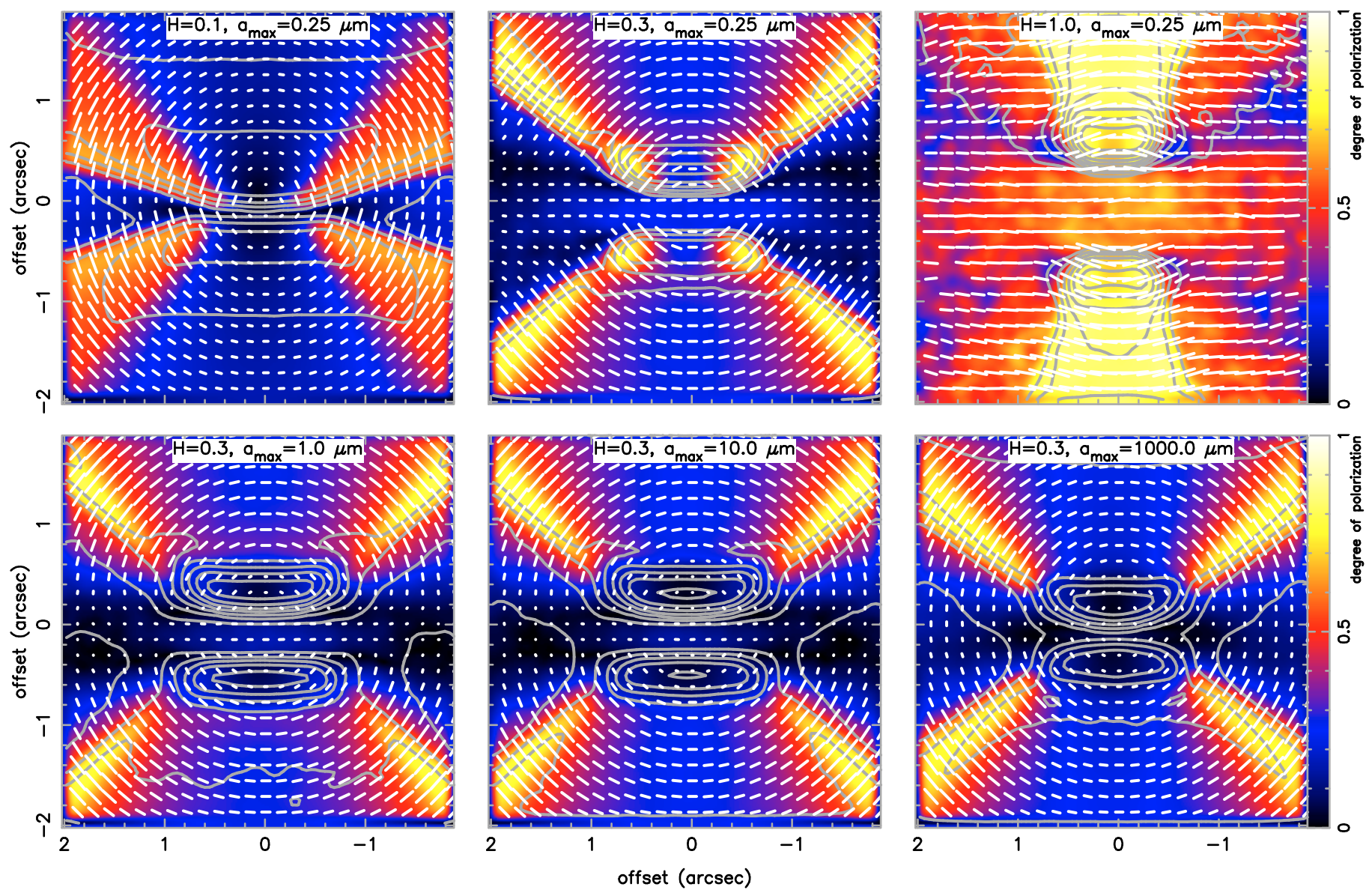

Fig. 2. A sample of our model results. The upper and bottom panels demonstrate the effects of the $H$ and $a_{\max }$ values, respectively. The inclination angle $\theta_{\mathrm{inc}}$ is $85^{\circ}$. The degree of linear polarization is indicated with the color scale, and the contour lines of the Stokes $I$ and the polarization vector lines are overlaid. The wavelength is $2.20 \mu \mathrm{m}$. The dust disk radius is 0.7 .

is throughly calibrated, for example, by using the coronagraphic technique or the PSF subtraction. With respect to the polarization properties towards the central star, since the observer sees the star more directly in near pole-on view, the polarization values are expected to express the disk information poorly. Thus, we focus on only edge-on $\left(\theta_{\text {inc }}=85^{\circ}\right)$ cases in later discussion.

We go on to investigate the wavelength dependence on the polarization. Figure 5 shows a plot of the polarization as a function of the wavelength $P(\lambda)$ from the $B$ to $K$ bands, which is derived with the same aperture of 0.2 in diameter. The sample models have an $H$ of $0.1,0.3$, and 1.0 and an $a_{\max }$ of 0.25 and $1000.0 \mu \mathrm{m}$. The $P(\lambda)$ has comparably constant values of $20 \%$ to $40 \%$ in the optical, while low $(\sim 1 \%)$ to high $(\sim 80 \%)$ values are obtained in the NIR. In the cases of large grains or low $H$ values, the $P(\lambda)$ peaks around a wavelength of $1.0 \mu \mathrm{m}$ and smoothly decreases with both longer and shorter wavelengths. Such a wavelength dependence is similar to the Serkowski law (Serkowski et al. 1975) and the Wilking law (Wilking et al. 1982) and is often found in the aperture polarimetry of T Tauri stars (e.g. Hodapp 1984; McGreor et al. 1994; Messinger et al. 1997; Whittet et al. 2001; Pereyra et al. 2009). We would like to point out that Serkowski's and Wilking's laws are intended to fit the interstellar polarization, where the polarization is produced by dichroic extinction, and the above similarity is met by chance. In contrast, flat and opposed behaviors of $P(\lambda)$ are found in models with high $H$ values and small grains, e.g., the models with $H=1.0$ and 0.3 and $a_{\max }=0.25 \mu \mathrm{m}$ and in some lowmass protostars (e.g. Lucas \& Roche 1998). This anti-law can

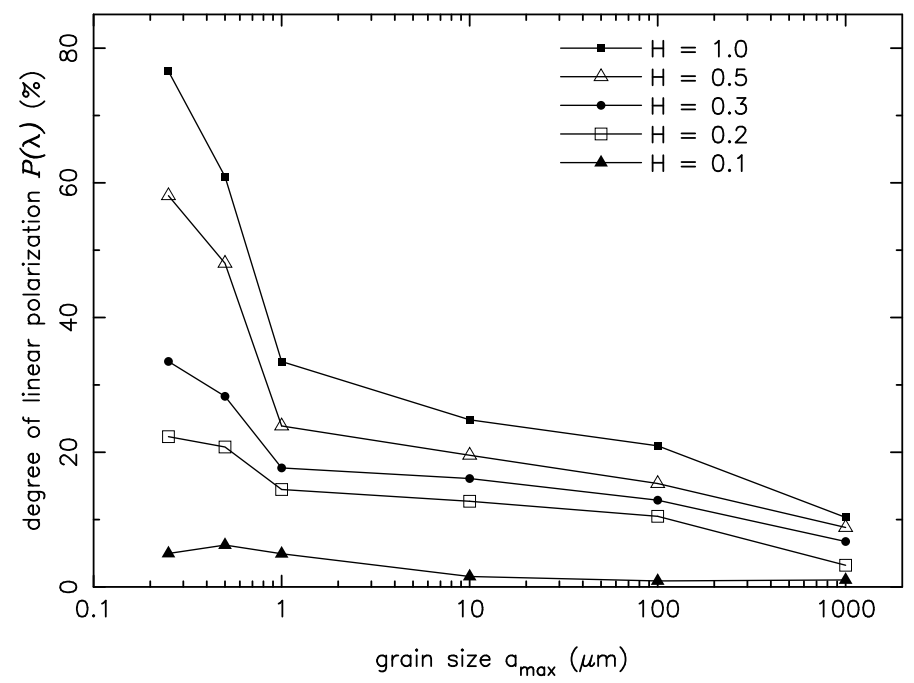

Fig. 3. Aperture polarimetry as a function of the grain size $a_{\max }$ at a wavelength of $2.20 \mu \mathrm{m}$. The results of $H=0.1,0.2,0.3,0.5$, and 1.0 are plotted. The aperture size is 0 !' 2 in diameter.

be explained without nonspherical grains, although we do not deny the effect of dichroism.

In the factors described above, only grain size has been discussed in previous papers (e.g. Hodapp 1984; Ménard et al. 1988). With scattering properties presented in Fig. 1a, it is easy to understand. Unfortunately, with the BM mechanism, it is hard 
K. Murakawa: High-resolution polarization disk
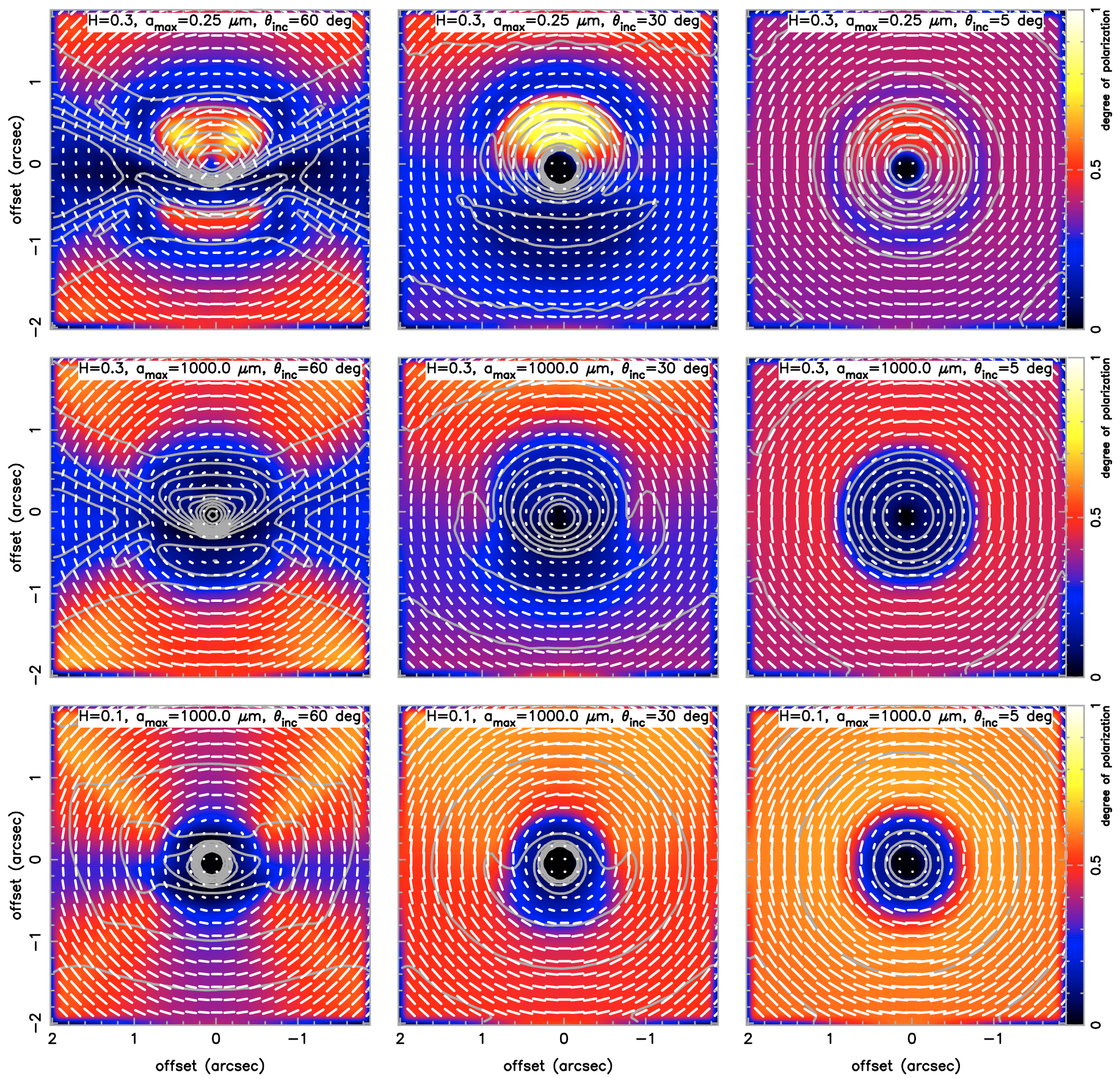

Fig. 4. Effects of inclination angle. The left, middle, and right panels show the results with inclination angles $\theta_{\text {inc }}=60^{\circ}, 30^{\circ}$, and $5^{\circ}$, respectively. The lower side of the nebulosity is tilted towards the observer. The results in Fig. 2 correspond to an edge-on case of $\theta_{\text {inc }}=85^{\circ}$. From top to bottom, the $H$ and $a_{\max }$ are $(0.3,0.25),(0.3,1000.0)$, and $(0.1,1000.0)$, respectively. The wavelength is $2.20 \mu \mathrm{m}$.

to explain the effects of the disk height and the wavelength dependence, because this theory is designed to explain the vector alignment. Therefore, we go back to the fundamental physics of the scattering model and develop a generalized interpretation to produce a polarization disk.

\section{Generalized scattering model}

When a photon emitted from the central star scatters for the first time, it is polarized. In the subsequent scattering event, the polarization orientation and the degree of polarization are changed. Every time the photon scatters or smiply passes through the dusty environment, the photon weight decreases. The flux towards a particular direction is the sum of photons that had different random walk histories, determining the final polarization status. We show some instructive models to see how this behavior appears in the polarization map in Fig. 6. The parameters have $H=0.3$ and $a_{\max }=0.25 \mu \mathrm{m}$, which are the same as the ones shown in the top middle panel of Fig. 2. However, the maximum number of scattering event $N_{\text {sca }}$ is limited to 1 (left) and 2 (right), which corresponds to single scattering and up to double scattering (cf. the $N_{\text {sca }}=50$ for results shown in Fig. 2). In the single scattering case, we see only a centro-symmetric vector pattern in the projected polar cavity down to the central 


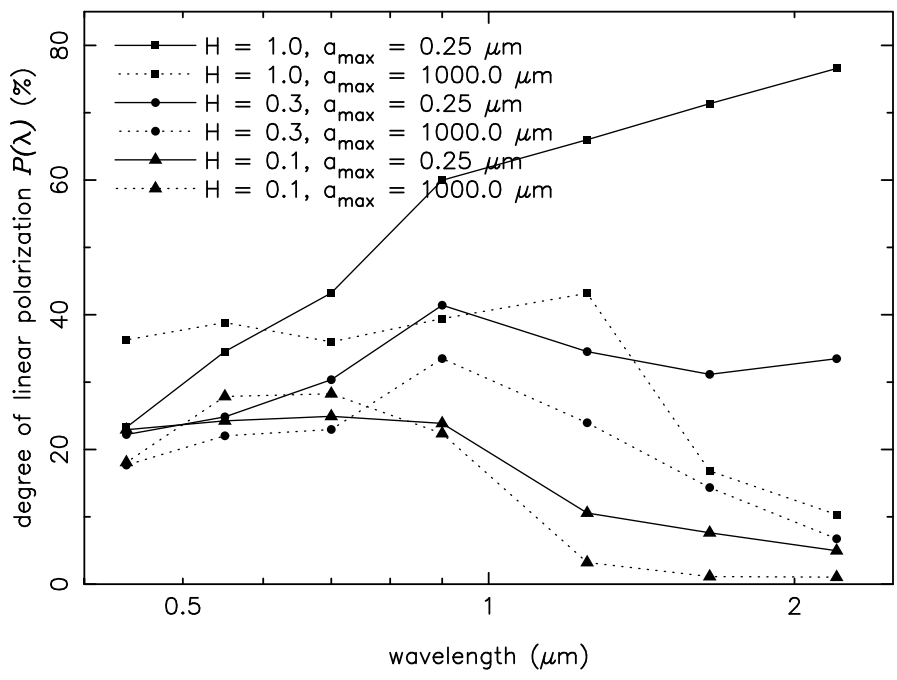

Fig. 5. Aperture polarimetry to show the wavelength dependence. The models with different $H$ values of $0.1,0.3$, and 1.0 and $a_{\max }$ values of 0.25 and $1000.0 \mu \mathrm{m}$ are plotted. The aperture size is 0.2 in diameter.

star. It is the disk surface from where the scattered light contributes the most flux. The photon does not reach from the disk on both sides of the equatorial plane, i.e., an equatorial region where the optical depth is too high $\left(\tau=6 \times 10^{4}\right.$ at $K$ band). The polarization is higher along the projected cavity boundary than the projected inner part of the cavity. This is explained with the scattering angle. The scattering angle is close to $\theta_{\max }$ $\left(\sim 90^{\circ}\right)$ in the projected cavity boundary (i.e. the disk surface), while the scattering angle of light from the projected inner part of the cavity approaches 0 (front side) and $180^{\circ}$ (rear side). In the double-scattering case, the centro-symmetric vector pattern is not seen towards the polarization disk. In this regime, a photon, that takes a path of a comparably lower extinction (e.g. disk surface) by avoiding a high density, equatorial region dominates the flux. We call this phenomenon the roundabout effect. Thanks to this effect, photons are even detected in regions where the single-scattered photons do not reach, and the appearance of a polarization disk does not directly trace the real dust disk, particularly in the equatorial direction in general. We would like to recall the definition of a polarization disk. Following the original $\mathrm{BM}$ idea, the polarization disk is a region towards the central star where the vectors are aligned along the equatorial direction. In Fig. 2, polarization disks are reproduced in results except the $H=0.1$ and $a_{\max }=0.25 \mu \mathrm{m}$ (top left panel) and the $H=0.3$ and $a_{\max }=1000.0 \mu \mathrm{m}$ (bottom right panel) models. In the last two exceptions, the negative detection is caused by a too thin dark lane and too low polarization, respectively. In both cases, a region with low polarizations exists in the immediate surroundings of the polarization disk. This region does not contain enough disk information to determine the disk parameters. Thus, this feature should be distinguished from a polarization disk, and "polarization dark lane" or "polarization shadow" is more suitable. For the final polarization status, although it is difficult to state rigorously because this is determined by various combinations of the geometrical configuration, a rough explanation is as follows. In the first scattering, the polarized component parallel to the equatorial direction exceeds the orthogonal component. Summing up the double-scattered light alters the polarization, there is more of the parallel component remaining than of the orthogonal one. This results in the vector alignment, which is, in essence, identical to the BM mechanism in this type of disk. Comparing the results between the different maximum number of scattering times, the double-scattered image is significantly different from the single-scattered image but is similar to the multiple-scattered image. The effect of the double scattering is the strongest in the polarization image in the $\mathrm{T}$ Tauri disk. However, according to the above logic, the net degree of polarization still more or less includes information on the first scattering event.

Our generalized theory can explain our model results, which are presented in Sect.2.2, and provide interpretations of some observations even not directly related to polarization or T Tauri disks. For the effect of the disk height, the $H$ value controls the scattering angle on the disk surface. A higher disk height causes the disk surface to be closer to the polar axis, where the scattering angle is closer to $\theta_{\max }$. The wavelength dependence is a tricky problem. In the NIR, since the difference in the grain sizes in the disk appears in the aperture polarimetry, the photons passing through the disk contribute to the flux. On the other hand, in the optical, the region where the photons stay away is larger, and the roundabout effect is more stronger than the NIR. The flux towards the polarization disk is dominated by the stray light going around the disk. Thus, the polarizations are characterized only by small grains in the envelope.

This roundabout effect also appears in some other observational methods. One is $3 \mu \mathrm{m}$ spectropolarimetry. In most objects, the polarization is enhanced in the water-ice absorption feature (e.g. Hough et al. 1989; Holloway et al. 2002). They interpreted this result as coming from an enhancement of dichroic absorption in this feature or a complex chemical composition of ice mantle around the dust core in the line of sight. We find that another explanation is possible, if we assume that grains in the disk are larger than in the envelope. In the water-ice absorption feature, the extinction is enhanced, causing the roundabout effect to also become stronger. As a result, higher polarization is produced by the grains in the disk surface or the envelope, whereas the polarization in the continuum is lower thanks to large grains in the disk. In addition, the $3 \mu \mathrm{m}$ absorption feature (the width and the position of the bottom of the feature) depends on the dust temperature and the grain size (e.g. Smith et al. 1989; Pendleton et al. 1990). If we assume Chiang \& Goldreich's double-layered disk (Chiang \& Goldreich 1997), we may derive the grain properties on the surface layer more than in the midplane. It would be possible to qualitatively estimate how much and where molecular formation progress by means of precise modeling.

We briefly comment on the nonspherical grain issue. The roundabout effect even works in disks with nonspherical grains. In cases of low polarization (e.g. $P<10 \%$ ) in the polarization disk, which are often detected in many $\mathrm{T}$ Tauri stars and Herbig Ae stars, it is not easy to find the effect of grain shape because grains are likely to be large, and the dichroism is weak. On the other hand, some massive stars have high NIR polarizations; e.g. G333.6 - 0.2; Fujiyoshi et al. (2001), S87; Chen et al. (2004), AFGL 437; Meakin et al. (2005), CRL 2136; Murakawa et al. (2008b), S255 IRS1; Simpson et al. (2009). The common feature is that the central star features are seen through the disk, and the polarization vectors are aligned along the disk plane. In the case of CRL 2136, we modeled the disk with only spherical grains with small sizes $\left(a_{\max }=0.45 \mu \mathrm{m}\right)$. The model result shows the centro-symmetric vector pattern, particularly in the $K$ band. The estimated optical depths of the disk in the $K$ band are 4.6 in the line of sight to the central star and 5.8 in the equatorial plane. In this intermediate optical depth of the disk, the single-scattered photons still reach the observer. Thus, the vector alignment is missing. Furthermore, the $H$-band image shows 


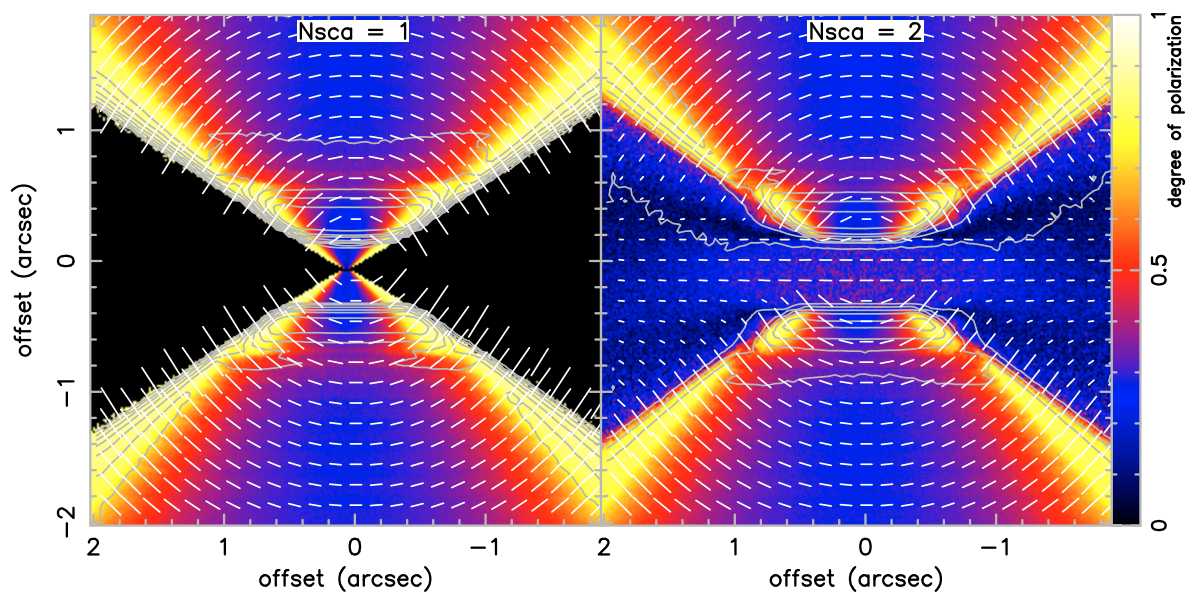

Fig. 6. The results of single-scattering (left $)$ and double-scattering images (right). The model parameters have $H=0.3$ and $a_{\max }=0.25 \mu \mathrm{m}$, which is the same as in the top middle panel in Fig. 2. The maximum number of scattering events $N_{\text {sca }}$ is limited to 1 (left) and 2 (right). The wavelength is $2.20 \mu \mathrm{m}$. A Gaussian function is not convolved. a spot-like region with an enhanced polarization at the central star IRS 1, but not elongated along the equatorial plane as seen in the polarization disk produced by the scattering models of the $\mathrm{T}$ Tauri disks. Objects with such a condition are the rule where the BM mechanism is weak. We speculate that they are one of promising samples to find the effects of aligned nonspherical grains.

\section{Conclusion}

We analyzed linear polarization maps of a sample of edge-on low-mass YSO disk models in the optical to the NIR in order to investigate the effects of the disk geometry and the grain sizes on the polarization disk properties. Our modeling treated scattering by spherical grains, aiming to develop a generalized scattering model to produce a polarization disk. This is an extension of the BM mechanism, which focuses on the vector alignment in the polarization disk. The point of our new interpretation is that multiply-scattered photons pass through a comparably low optical depth region (e.g. the disk surface) as if they were avoiding the high-density, equatorial region in the disk, which we call the roundabout effect. Although the single-scattered light does not directly reach the observer, and the double-scattered light dominates the flux most, the degree of polarization still possesses information on the first scattering event, i.e. the geometrical configuration of the disk surface.

Our model examined various disk heights $(H=0.1$ to 1.0) and various grain sizes in the disk $\left(a_{\max }=0.25 \mu \mathrm{m}\right.$ to $1000.0 \mu \mathrm{m})$, which are important parameters on the dust settling and grain growth in the disk. In the pole-on view, the polarization vectors towards the disk are centro-symmetrically aligned. This polarization property is determined by the geometrical configuration of the disk surface and the grain sizes. If the unpolarized flux from the central star is properly subtracted, the pole-on disk can be distinguished from the outer envelope. On the other hand, the polarization properties, such as the degree of polarization and vector alignment, appear in the polariation disk. Models with high $H$ values $(H=1.0)$ and small grains $\left(a_{\max }=0.25 \mu \mathrm{m}\right)$ produce high polarization (up to $\sim 80 \%$ in the $K$ band), and other conditions cause low polarizations. The latter is due to the grain size dependence on the polarization. The former is explained by the scattering angle dependence in our new model. We also investigated the wavelength dependence of the polarization $P(\lambda)$. The NIR polarization has variety, while it is comparably constant $(20-40 \%)$ in the optical, where only the photons passed from the envelope are observed because of the roundabout effect. In contrast, the NIR photons that interacted with dust in the disk still reach the observer. In other words, NIR polarimetry may offer a chance to diagnose the dust disk, but it is probably hopeless in the optical in edge-on YSO cases. In addition, Serkowski's law-like behavior is obtained in models with low $H$ values or large grains, which are detected in many $\mathrm{T}$ Tauri stars. When the grains are small and the disk height is large, which is seen in some low-mass protostars, the opposite, or flat, wavelength dependence is reproduced. This is also explained with our new model, although we do not intend to completely rule out the effects of dichroism by nonspherical grains.

We would like to stress that the roundabout effect must occur even in nonspherical grain models. This means that the vector alignment is produced not only by dichroic absorption but also by (dichroic) scattering. We may detect the effect of dichroism in objects if the disk has an intermediate optical depth and the polarization vectors are aligned, because the BM and roundabout effects are weak in such objects.

\section{References}

Andrews, S. M., \& Williams, J. P. 2007, ApJ, 671, 1800

Bastien, P., \& Ménard, F. 1988, ApJ, 326, 334 (BM)

Bastien, P., \& Ménard, F. 1990, ApJ, 364, 232

Beckford, A. F., Lucas, P. W., Chrysostomou, A. C., \& Gledhill, T. M. 2008, MNRAS, 384, 907

Beckwith, S. V. W., Sargent, A. I., Chini, R. S., \& Guesten, R. 1990, AJ, 99, 924 Bohren, C. F., \& Huffmann, D. R., 1983, Absorption and scattering of light by small particles (New York: John Wiley \& Sons)

Cassen, P., \& Moosman, A. 1981, Icarus, 48, 353

Chen, X., Yao, Y., Yang, J., Jiang, Z., \& Ishii, M. 2004, A\&A, 428, 523

Chiang, E. I., \& Goldreich, P. 1997, ApJ, 490, 368

Draine, B. T., \& Lee, H. M. 1984, ApJ, 285, 89

Fischer, O., Henning, Th., \& Yorke, H. W. 1994, A\&A, 284, 187

Fischer, O., Henning, Th., \& Yorke, H. W. 1996, A\&A, 308, 863

Fujiyoshi, T., Smith, C. H., Wright, C. M., et al., 2001, MNRAS, 327, 233

Fujiyoshi, T., et al. 2008, Astronomical Polarimetry 2008

Hodapp, K.-H. 1984, A\&A, 141, 255

Holloway, R. P., Chrysostomou, A., Aitken, D. K., Hough, J. H., \& McCall, A. 2002, MNRAS, 336, 425

Hough, J. H., Whittet, D. C. B., Sato, S., et al. 1989, MNRAS, 241, 71

Jiang, Z., Tamura, M., Hoare, M. G., et al. 2008, ApJ, 673, L175

Kenyon, S. J., Whitney, B. A., Gomez, M., \& Hartmann, L. 1993, ApJ, 414, 773 Koenigl, A. 1991, ApJ, 370, 39

Lucas, P. W., \& Roche, P. F. 1997, MNRAS, 286, 895

Lucas, P. W., \& Roche, P. F. 1998, MNRAS, 299, 699

Lucas, P. W., Fukagawa, M., Tamura, M., et al. 2004, MNRAS, 352, 1347

McGregor, P. J., Harrison, T. E., Hough, J. H., \& Bailey, J. A. 1994, MNRAS, 267,755 
A\&A 518, A63 (2010)

Mathis, J. S., Rumple, W., \& Nordsieck, K. H. 1977, ApJ, 217, 425 Meakin, C. A., Hines, D. C., \& Thompson, R. I. 2005, ApJ, 634, 1146 Ménard, F., Bastien, P., \& Roberts, C. 1988, ApJ, 335, 290

Messinger, D. W., Whittet, D. C. B., \& Roberge, W. G. 1997, ApJ, 487, 314 Mestel, L., \& Paris, R. B. 1984, A\&A, 136, 98

Monin, J.-L., \& Bouvier, J. 2000, A\&A, 356, 75

Murakawa, K., Preibisch, T., Kraus, S., et al. 2008a, A\&A, 488, L75

Murakawa, K., Preibisch, T., Kraus, S., \& Weigelt, G. 2008b, A\&A, 490, 673

Murakawa, K., Oya, S., Pyo, T.-S., \& Ishii, M. 2008c, A\&A, 492, 731

Ostriker, E. C., \& Shu, Frank H. 1995, ApJ, 447, 813

Pendleton, Y. J., Tielens, A. G. G. M., \& Werner, M. W. 1990, ApJ, 349, 107

Pereyra, A., Girart, J. M., Magalháes, A. M., et al. 2009, A\&A, 501, 595

Perrin, M. D., Vacca, W. D., \& Graham, J. R. 2009, AJ, 137, 4468

Piirola, V., Scaltriti, F., \& Coyne, G. V. 1992, Nature, 359, 339

Pringle, J. E. 1981, ARA\&A, 19, 137

Serkowski, K., Mathewson, D. S., \& Ford, V. L. 1975, ApJ, 196, 261
Shakura, N. I., \& Sunyaev, R. A. 1973, A\&A, 24, 337

Simpson, J. P., Burton, M. G., Colgan, S. W. J., et al. 2009, ApJ, 700, 1488

Smith, R. G., Sellgren, K., \& Tokunaga, A. T. 1989, ApJ, 344, 413

Stapelfeldt, K. R., Krist, J. E., Menard, F., et al. 1998, ApJ, 502, 65

Terebey, S., Shu, F. H., \& Cassen, P. 1984, ApJ, 286, 529

Ulrich, R. K. 1976, ApJ, 210, 377

Warren-Smith, R. F., Draper, P. W., \& Scarrott, S. M. 1987, MNRAS, 227, 749

Watson, A. M., \& Stapelfeldt, K. R. 2007, AJ, 133, 845

Whitney, B. A., \& Hartman, L. 1993, ApJ, 402, 605

Whitney, B. A., \& Wolff, M. J. 2002, ApJ, 574, 205

Whitney, B. A., Kenyon, S. L., \& Gomez, M. 1997, ApJ, 485, 703

Whitney, B. A., Wood, K, Bjorkman, J. E., \& Cohen, M. 2003, ApJ, 598, 1079

Whittet, D. C. B., Pendleton, Y. J., Gibb, E. L., et al. 2001, ApJ, 550, 793

Wilking, B. A., Lebofsky, M. J., \& Rieke, G. H. 1982, AJ, 87, 695

Wolf, S., Voshchinnikov, N. V., \& Henning, Th. 2002, A\&A, 385, 365

Wood, K., Kenyon, S. J., Whitney, B., \& Turnbull, M. 1998, ApJ, 497, 404 